\title{
Ligand-Field Calculations on Pseudo-Tetragonal Ni(II) Compounds
}

\author{
Interpretation of Magnetic Measurements and Ligand-field Spectra in a Single Model \\ A. Vermaas and W. L. Groeneveld \\ Department of Coordination Chemistry, Gorlaeus Laboratories, State University, \\ P.O. Box 75, Leiden, The Netherlands \\ and J. Reedijk \\ Department of Chemistry, Delft University of Technology, Delft, The Netherlands \\ (Z. Naturforsch. 32 a, 632-640 [1977] ; received March 28, 1977)
}

\begin{abstract}
Magnetic susceptibility, magnetic saturation, ligand field and EPR measurements are reported for the compounds $\mathrm{Ni}(5 \text {-methyl pyrazole })_{4} \mathrm{X}_{2},(\mathrm{X}=\mathrm{Cl}, \mathrm{Br}, \mathrm{I})$. All data are interpreted using ligand-field calculations, yielding parameters $D q, D s, D t, B, C, g_{\|}, g_{\perp}, D, \zeta_{\|}, \zeta_{\perp}$ and $k$.

All data fit with a single set of parameters. The zero-field splittings and $g$ values are determined from the magnetic measurements, whereas the excited state splittings are determined from the ligand-field spectra.

In studying the relation between the zero-field splitting of the $\mathrm{Ni}$ (II) ion and both the spin. orbit coupling and spectroscopic energy parameters, a description using an anisotropic spin-orbit coupling gave the most reliable fit.

In addition, known ligand-field spectra and $D$ values of $\mathrm{Ni}$ (pyrazole) ${ }_{4} \mathrm{X}_{2},(\mathrm{X}=\mathrm{Cl}, \mathrm{Br}, \mathrm{I})$ were considered. The derived energy parameters of the corresponding pyrazole and 5-methyl pyrazole compounds are very similar.
\end{abstract}

\section{Introduction}

Nickel(II) ions in octahedral symmetry posses a ${ }^{3} \mathrm{~A}_{2 \mathrm{~g}}$ ground state and three threefold orbitally degenerate excited states with spin multiplicity three. When the symmetry is lowered, the threefold spindegenerate ground state splits by second-order spinorbit interaction with the excited states that exhibit an orbital splitting ${ }^{1}$. Therefore, the magnitude of the ground state splitting parameter(s) will depend upon the orbital splittings of the excited states.

In the past Gerloch et al. ${ }^{2}$ have calculated crystalfield and Racah parameters from experimental data, and calculated the zero-field splitting in a tetragonal case for sets of crystal-field and Racah parameters; however, no measurements to obtain the zero-field splitting for the compounds under investigation were reported. Several other workers ${ }^{3-6}$ also calculated crystal-field and Racah parameters from their optical measurements.

In order to determine the relation between the ground state splitting and the orbital splittings of the excited states, we determined both the ground state splittings and the energies of the excited states for three compounds with formula $\mathrm{Ni}(\mathrm{mpz})_{4} \mathrm{X}_{2}$,

Reprint requests to Dr. A. Vermaas, Gorlaeus Lab. k 414, State University, P.O. Box 75 , Leiden/Netherlands. where $\mathrm{mpz}$ stands for 5 -methyl pyrazole and $\mathrm{X}=\mathrm{Cl}$, $\mathrm{Br}$ or I. In addition, earlier measurements on $\mathrm{Ni}(\mathrm{pz}){ }_{4} \mathrm{X}_{2}$ that have relevance to this subject were reinvestigated. In this formula $\mathrm{pz}$ stands for pyrazole. The magnitude of the ground-state splitting was determined by magnetization measurements and EPR spectra, and the energies of the excited states were obtained from diffuse reflectance spectra.

\section{Experimental Section}

The compounds $\mathrm{Ni}(\mathrm{mpz}){ }_{4} \mathrm{X}_{2}$ have been synthesized as described previously ${ }^{7}$. Magnetization measurements of powdered samples were performed by means of a PAR vibrating sample magnetometer, model 150. For each compound both magnetic saturation from 0 to 52,400 Oersted at distinct temperatures, and magnetic susceptibility in the 2 to $80 \mathrm{~K}$ region at fields of about 5000 Oersted were measured.

Diffuse reflectance spectra were obtained with a Beckman DK-2A spectrophotometer in the 325 to $2000 \mathrm{~nm}$ region, using magnesium oxide as a reference. A low temperature attachment was used, as described by Fackler and Holah ${ }^{8}$.

Paramagnetic resonance spectra of powdered samples were recorded both at X-band and Q-band frequencies on Varian instruments by methods described previously 9,10 . 


\section{Starting Points and Theory}

\section{Model}

Discussing the relation between the ground state splitting and the energies of the excited states, we will use calculations that involve the diagonalization of the complete $\mathrm{d}^{8}$ manifold, perturbed by interelectronic repulsion, crystal-field and spin-orbit coupling effects and the Trees correction ${ }^{11-13}$ under the Hamiltonian.

$$
\hat{H}=\sum_{i<j}^{2} e^{2} / r_{i j}+\hat{V}_{\text {c.f. }}+\hat{H}_{\text {s.o. }}+\alpha L(L+1)
$$

\section{Structural Details}

To set up an expression for the crystal-field Hamiltonian, we used known crystal structures of pyrazole and 5-methylpyrazole compounds. The crystal structures of $\mathrm{Ni}(\mathrm{pz})_{4} \mathrm{Cl}_{2}$ and $\mathrm{Ni}(\mathrm{pz})_{4} \mathrm{Br}_{2}$ have been published ${ }^{14,15}$. These crystals are both monoclinic and have space group C2/c with four molecules per unit cell. The only rigorous molecular symmetry element in these molecules is a centre of symmetry on the nickel ion, and thus the rigorous point group is $\mathrm{C}_{\mathrm{i}}$. For the $\mathrm{Ni}(\mathrm{N}){ }_{4} \mathrm{X}_{2}$ unit, however, the point symmetry appears to be close to $D_{4 h}$ : the $\mathrm{Ni}-\mathrm{N}$ distances of the two types of different pyrazole rings are nearly the same, and the deviation of the $\mathrm{X}-\mathrm{Ni}-\mathrm{X}$ line with the normal to the plane through the four coordinating nitrogen atoms (the basal plane) is less than 0.5 degrees. For the $\mathrm{Ni}(\mathrm{pz})_{4} \mathrm{X}_{2}$ unit, the pyrazole rings are only slightly tilted from a position perpendicular to the basal plane, so that an axis perpendicular to that plane is nearly a fourfold axis, as far as the arrangements of the atoms in the rings are not considered.

For the compounds $\mathrm{Ni}(\mathrm{mpz})_{4} \mathrm{X}_{2}$ no crystal structures are known. The crystal structure of $\mathrm{Mn}(\mathrm{mpz}){ }_{4} \mathrm{Br}_{2}{ }^{16}$, however, has been reported. The $\mathrm{X}$-ray diffraction patterns of the powders of $\mathrm{Mn}(\mathrm{mpz}){ }_{4} \mathrm{Br}_{2}$ and $\mathrm{Ni}(\mathrm{mpz}){ }_{4} \mathrm{Br}_{2}$ appeared to be very similar both in $d$ values and intensities ${ }^{7}$. Therefore, we assumed that the compounds $\mathrm{Ni}(\mathrm{mpz}){ }_{4} \mathrm{X}_{2}$ have the same crystal and molecular structure as $\mathrm{Mn}(\mathrm{mpz})_{4} \mathrm{Br}_{2}$. This latter compound has space group $\mathrm{P} \overline{\mathrm{I}}$ with one molecule per unit cell. The only rigorous symmetry element is a centre of symmetry on the $\mathrm{Mn}$ (II) ion and thus the rigorous point group is $\mathrm{C}_{\mathrm{i}}$. However, the point symmetry appears to be close to $\mathrm{C}_{2 \mathrm{~h}}$. As in the pyrazole compounds, the
$\mathrm{Mn}-\mathrm{N}$ distances of the two types of different pyrazole rings are nearly the same and, furthermore, the deviation of the $\mathrm{Br}-\mathrm{Mn}-\mathrm{Br}$ line from the normal to the plane through the four coordinating nitrogens (the basal plane) is less than 1.5 degrees. Contrary to the pyrazole compounds however, the methyl pyrazole rings are tilted much more from a position perpendicular to the basal plane. The values of the tilt angles for the two rings are nearly equal. The consequence of this feature is that, besides the rigorous symmetry element of inversion, the only pseudo-symmetry elements are a twofold axis in the basal plane, and a plane of symmetry perpendicular to that axis. This results in a point symmetry close to $\mathrm{C}_{2 \mathrm{~h}}$. We assume in the following sections that the chloride and iodide compounds have similar point symmetry.

\section{Calculations}

The crystal-field Hamiltonian we have chosen for the pyrazole compounds is one that agrees with the point group $\mathrm{D}_{4 \mathrm{~h}}$. With the aid of the method given by Hutchings ${ }^{17}$ and after evaluating the radial parts of the matrix elements a one-electron crystal-field Hamiltonian is obtained that is acting on the angular parts of the wave functions:

$$
\begin{aligned}
V_{\text {c.f. }}^{\prime}= & D q^{x y} \cdot\left(\mathrm{O}_{4}{ }^{0}+5 \cdot \mathrm{O}_{4}^{4}\right) / 12+D s \cdot \mathrm{O}_{2}{ }^{0} / 3 \\
& -D t \cdot \mathrm{O}_{4}{ }^{0} / 12
\end{aligned}
$$

where $D q^{x y}, D s$ and $D t$ have their usual meaning ${ }^{1}$.

The theory of spin-orbit coupling effects in crystal fields of lower symmetry has been thoroughly investigated by Lulek ${ }^{18}$.

For our purpose the spin-orbit coupling Hamiltonian is simplified to

$$
\hat{H}_{\mathrm{s} .0 .}=-\sum_{i=1}^{2}\left[\zeta_{\|} 1_{i z} s_{i z}+\zeta_{\perp}\left(1_{i x} s_{i x}+1_{i y} s_{i y}\right)\right]
$$

where $\zeta_{\|}$and $\zeta_{\perp}$ are the spin-orbit coupling parameters.

By the action of the complete $\mathrm{D}_{4 \mathrm{~h}}$ perturbation Hamiltonian, the ground state which is ${ }^{3} \mathrm{~A}_{2 g}$ in an octahedron, splits into a non-degenerate and a twofold degenerate level. Concerning the excited states, the three orbital triplet states in octahedral field, all split into two states if spin-orbit coupling effects are neglected. This splitting has been observed in the polarized single crystal spectra for the two excited triplet states that are lowest in energy ${ }^{6}$. 
The diffuse reflectance spectra of the 5-methyl pyrazole compounds are very similar to those of the pyrazole compounds. The additional splitting of excited states by the descending of symmetry from point group $\mathrm{D}_{4 \mathrm{~h}}$ to $\mathrm{C}_{2 \mathrm{~h}}$ could not be detected, being apparently too small with respect to the experimental resolution.

Since the primary purpose of this investigation is an interpretation of both optical and magnetic measurements within the same model, a perturbation Hamiltonian appropriate to $\mathrm{D}_{4 \mathrm{~h}}$ symmetry, i.e.

$$
H=\sum_{i<j}^{2} e^{2} / r_{i j}-\sum_{i=1}^{2} V_{\text {e.f. }}^{\prime}(i)+H_{\text {s.o. }}+\alpha L(L+1)
$$

will also be used for the mpz compounds. In (4) the summation over $i$ is the summation over the two electron holes.

Calculated matrix elements of the $45 \times 45$ matrix for a $D_{4 h}$ Hamiltonian with isotropic spin-orbit interaction have been reported before ${ }^{20,21}$ : Perumareddi ${ }^{20}$ used a set of symmetry adapted eigenvectors; Mooney and Smith ${ }^{21}$ used a set of basic functions in $\left|L, L_{z}, S, S_{z}\right\rangle$ quantization. However, some of the matrix elements used by Mooney and Smith are incorrectly printed.

The correct expressions for these matrix elements are:

$$
\begin{aligned}
A(2,11) & =A(8,29)=\zeta / 2 \\
A(12,12) & =A(28,28) \\
& =-3 D q-3 \zeta / 2+D s+3 D t+12 \alpha, \\
A(4,19) & =A(6,21)=5 \zeta / \sqrt{70} \\
A(16,16) & =A(24,24) \\
& =-D q+\zeta / 2-3 D s / 5+D t+12 \alpha, \\
A(19,19) & =A(20,20)=A(21,21) \\
& =-6 D q-4 D s / 5+6 D t+12 \alpha, \\
A(19,32) & =A(21,34)=-\sqrt{(12 / 35) \zeta}, \\
A(45,45) & =22 B+7 C
\end{aligned}
$$

\section{Results and Discussion}

\section{Ligand-field Spectra and Calculations Concerning Excited State Energies}

Room temperature ligand-field spectra of $\mathrm{Ni}(\mathrm{mpz})_{4} \mathrm{X}_{2}$ where $\mathrm{X}$ is a halide have been published before ${ }^{19}$. We have now also measured the diffuse reflectance spectra at lower temperature. The shapes of these spectra are very similar to those published before. The spectra show small shifts to higher energy as usually observed upon cooling. The absorption bands have been fit with values calculated with the Hamiltonian in (4). The assignment of the peaks was taken analogous to that in the pz compounds ${ }^{6}$.

Furthermore, some transitions in the $20,000 \mathrm{~cm}^{-1}$ region could be assigned to triplet-singlet transitions. These transitions were not assigned by Reimann ${ }^{6}$. Using these assignments we are able to calculate the value of the Racah parameter $C$.

The observed absorption bands as well as the calculated values are presented in Table I. For the experimental absorption bands of $\mathrm{Ni}(\mathrm{pz})_{4} \mathrm{Cl}_{2}$ and $\mathrm{Ni}(\mathrm{pz}){ }_{4} \mathrm{Br}_{2}$ we used the data given by Reimann ${ }^{6}$. Because of the resolution of our spectrophotometer and band broadening due to vibrations, only band splittings larger than about $500 \mathrm{~cm}^{-1}$ could be detected, so that no spin-orbit splittings could be detected. The reported calculated values are the average values over a number of levels originating from the same orbital terms and are rounded on multiples of $100 \mathrm{~cm}^{-1}$.

The spectrochemical parameters that give the best fit within our assignment are listed in Table II. In the calculations, the spin-orbit coupling parameters were chosen such that they are in agreement with the zero field splitting (vide infra) and $\alpha$ was fixed at $75 \mathrm{~cm}^{-1}$. This value of $\alpha$ means a reduction with respect to the free ion value as is usually observed in nickel (II) compounds ${ }^{21}$. Neglecting the Trees correction (thus, $\alpha=0$ ) yields poorer fits. In that case the calculated $\mathrm{B}$ values are in general $50 \mathrm{~cm}^{-1}$ smaller.

Concerning the values of the crystal field parameters the following remarks can be made:

i. The $D q^{r y}$ values are all very close to each other; in agreement with the proposed structure the $D q^{x y}$ of the pyrazole compounds is nearly the same as that of the corresponding 5-methyl pyrazole compounds. The $D q^{x y}$ values increase with decreasing temperature, except for $\mathrm{Ni}(\mathrm{mpz})_{4} \mathrm{I}_{2}$. The increase in $D q^{x y}$ is a feature that is generally observed $^{6,22}$ and is thought to be caused by the compression of the crystal with decreasing temperature. The deviating behaviour of $\mathrm{Ni}(\mathrm{mpz}){ }_{4} \mathrm{I}_{2}$ may be due to the fact that the two absorptions near 11,000 $\mathrm{cm}^{-1}$ could not be detected separately and, furthermore, the spin-allowed band highest in energy was not observed because of a charge transfer absorption in that region. 
Table I. Observed and calculated ligand-field transitions of $\mathrm{Ni}$ (II) compounds.

\begin{tabular}{|c|c|c|c|c|c|c|c|c|c|}
\hline \multirow{3}{*}{$\begin{array}{l}\text { Compound } \\
\text { Temperature (K) } \\
\text { Absorption } \\
\text { frequencies }\left(\mathrm{cm}^{-1}\right)\end{array}$} & \multirow{2}{*}{\multicolumn{2}{|c|}{$\frac{\mathrm{Ni}(\mathrm{pz}){ }_{4} \mathrm{Cl}_{2}}{295}$}} & \multirow{2}{*}{\multicolumn{2}{|c|}{$\frac{\mathrm{Ni}(\mathrm{pz})_{4} \mathrm{Br}_{2}}{295}$}} & \multicolumn{4}{|c|}{$\mathrm{Ni}(\mathrm{mpz}){ }_{4} \mathrm{Cl}_{2}$} & \multirow[t]{3}{*}{ Assignment a } \\
\hline & & & & & \multicolumn{2}{|l|}{110} & \multicolumn{2}{|l|}{295} & \\
\hline & obs. & calc. & obs. & calc. & obs. & calc. & obs. & calc. & \\
\hline & 8000 & 8000 & 7240 & 7250 & 8100 & 8100 & 7900 & 7900 & ${ }^{3} \mathrm{E}_{\mathrm{g}}\left({ }^{3} \mathrm{~T}_{2 \mathrm{~g}}(\mathrm{~F})\right) \leftarrow{ }^{3} \mathrm{~B}_{1 \mathrm{~g}}$ \\
\hline & 10950 & 10950 & 10900 & 10900 & 11100 & 11100 & 11000 & 11000 & ${ }^{3} B_{2 g}\left({ }^{3} T_{2 g}(F)\right) \leftarrow$ \\
\hline & 13100 & 13100 & 12000 & 12000 & 13300 & 13300 & 13100 & 13100 & ${ }^{3} \mathrm{~A}_{2 \mathrm{~g}}\left({ }^{3} \mathrm{~T}_{1 \mathrm{~g}}(\mathrm{~F})\right) \leftarrow$ \\
\hline & 13500 & 13700 & - & 13600 & - & 13600 & - & 13500 & ${ }^{1} \mathrm{~B}_{1 \mathrm{~g}}\left({ }^{1} \mathrm{E}(\mathrm{D})\right) \leftarrow$ \\
\hline & 16400 & 16400 & 15900 & 15900 & 16600 & 16500 & 16200 & 16200 & ${ }^{3} \mathrm{E}_{\mathrm{g}}\left({ }^{3} \mathrm{~T}_{1 \mathrm{~g}}(\mathrm{~F})\right) \leftarrow$ \\
\hline & $21000 *$ & 21000 & - & 20100 & 21100 & 21100 & 20800 & 20800 & ${ }^{1} \mathrm{E}_{\mathrm{g}}\left({ }^{1} \mathrm{~T}_{2 \mathrm{~g}}(\mathrm{D})\right) \leftarrow$ \\
\hline & $23200 *$ & 23800 & - & 23200 & 23400 & 23900 & - & 23700 & ${ }^{1} \mathrm{~B}_{2 \mathrm{~g}}\left({ }^{1} \mathrm{~T}_{2 \mathrm{~g}}(\mathrm{D})\right) \leftarrow$ \\
\hline & 26500 & 26500 & 25800 & 25800 & 26600 & 26600 & 25900 & 25900 & ${ }^{3} \mathrm{E}_{\mathrm{g}},{ }^{3} \mathrm{~A}_{2 \mathrm{~g}}\left({ }^{3} \mathrm{~T}_{1 \mathrm{~g}}(\mathrm{P})\right) \leftarrow$ \\
\hline Compound & \multicolumn{4}{|c|}{$\mathrm{Ni}(\mathrm{mpz}){ }_{4} \mathrm{Br}_{2}$} & \multicolumn{4}{|c|}{$\mathrm{Ni}(\mathrm{mpz}){ }_{4} \mathrm{I}_{2}$} & \multirow[t]{3}{*}{ Assignment a } \\
\hline Temperature (K) & \multicolumn{2}{|l|}{125} & \multicolumn{2}{|l|}{295} & \multicolumn{2}{|l|}{135} & \multicolumn{2}{|l|}{295} & \\
\hline \multirow[t]{9}{*}{$\begin{array}{l}\text { Absorption } \\
\text { frequencies }\left(\mathrm{cm}^{-1}\right)\end{array}$} & obs. & calc. & obs. & calc. & obs. & calc. & obs. & calc. & \\
\hline & 7500 & 7500 & 7400 & 7400 & 6500 & 6500 & 6200 & 6200 & ${ }^{3} \mathrm{E}_{\mathrm{g}}\left({ }^{3} \mathrm{~T}_{2 \mathrm{~g}}(\mathrm{~F})\right) \leftarrow{ }^{3} \mathrm{~B}_{1 \mathrm{~g}}$ \\
\hline & 11000 & 11000 & 10800 & 10800 & 10800 & 10800 & 10900 & 10900 & ${ }^{3} \mathrm{~B}_{2 \mathrm{~g}}\left({ }^{3} \mathrm{~T}_{2 \mathrm{~g}}(\mathrm{~F})\right) \leftarrow$ \\
\hline & 12100 & 12000 & 11900 & 11900 & - & - & - & - & ${ }^{3} \mathrm{~A}_{2 \mathrm{~g}}\left({ }^{3} \mathrm{~T}_{1 \mathrm{~g}}(\mathrm{~F})\right) \leftarrow$ \\
\hline & - & 13800 & - & 13400 & - & 13400 & - & 13500 & ${ }^{1} \mathrm{~B}_{1 \mathrm{~g}}\left({ }^{1} \mathrm{E}_{\mathrm{g}}(\mathrm{D})\right) \leftarrow$ \\
\hline & 16300 & 16300 & 15900 & 15900 & 15600 & 15500 & 15200 & 15200 & ${ }^{3} \mathrm{E}_{\mathrm{g}}\left({ }^{3} \mathrm{~T}_{1 \mathrm{~g}}(\mathrm{~F})\right) \leftarrow$ \\
\hline & 20500 & 20500 & 20000 & 20000 & 19200 & 19200 & 19000 & 19000 & ${ }^{1} \mathrm{E}_{\mathrm{g}}\left({ }^{1} \mathrm{~T}_{2 \mathrm{~g}}(\mathrm{D})\right) \leftarrow$ \\
\hline & - & 22600 & - & 22400 & - & - & - & - & ${ }^{1} \mathrm{~B}_{2 \mathrm{~g}}\left({ }^{1} \mathrm{~T}_{2 \mathrm{~g}}(\mathrm{D})\right) \leftarrow$ \\
\hline & 26100 & 26100 & 25500 & 25500 & - & - & - & - & ${ }^{3} \mathrm{E}_{\mathrm{g}},{ }^{3} \mathrm{~A}_{2 \mathrm{~g}}\left({ }^{3} \mathrm{~T}_{1 \mathrm{~g}}(\mathrm{P})\right) \leftarrow$ \\
\hline
\end{tabular}

* At $77 \mathrm{~K}^{0}$.

a The given representations are irreducible representations of the $\mathrm{D}_{4 \mathrm{~h}}$ point group. In parentheses are the representations of the splitted octahedral point group and the free ion multiplets.

\begin{tabular}{lcccccccc}
\hline Compound & $\begin{array}{c}\text { Temp. } \\
(\mathrm{K})\end{array}$ & $D q^{x y}$ & \multicolumn{1}{c}{$D s$} & $D t$ & $B$ & $C$ & $\alpha$ & $D q^{z}$ \\
\hline $\mathrm{Ni}(\mathrm{pz})_{4} \mathrm{Cl}_{2}$ & 295 & 1095 & 1040 & 285 & 940 & 3400 & 75 & 595 \\
$\mathrm{Ni}(\mathrm{pz})_{4} \mathrm{Br}_{2}$ & 295 & 1090 & 1250 & 345 & 920 & 3400 & 75 & 490 \\
$\mathrm{Ni}(\mathrm{mpz})_{4} \mathrm{Cl}_{2}$ & 110 & 1110 & 1000 & 290 & 935 & 3400 & 75 & 600 \\
$\mathrm{Ni}(\mathrm{mpz})_{4} \mathrm{Cl}_{2}$ & 295 & 1100 & 950 & 300 & 900 & 3450 & 75 & 580 \\
$\mathrm{Ni}(\mathrm{mpz})_{4} \mathrm{Br}_{2}$ & 125 & 1100 & 1350 & 320 & 905 & 3525 & 75 & 540 \\
$\mathrm{Ni}(\mathrm{mpz})_{4} \mathrm{Br}_{2}$ & 295 & 1080 & 1250 & 320 & 890 & 3400 & 75 & 520 \\
$\mathrm{Ni}(\mathrm{mpz})_{4} \mathrm{I}_{2}$ & 135 & 1080 & 1500 & 390 & 900 & 3400 & 75 & 400 \\
$\mathrm{Ni}(\mathrm{mpz})_{4} \mathrm{I}_{2}$ & 295 & 1090 & 1400 & 440 & 900 & 3400 & 75 & 320 \\
\hline
\end{tabular}

Table II.

Spectrochemical parameters of $\mathrm{Ni}$ (II) compounds (in $\mathrm{cm}^{-1}$ ). ii. The tetragonal distortion parameters $D s$ and $D t$ are large, indicating a large difference in coordinating properties between the pyrazole ligands and the halide ions.

iii. The $D s$ and $D t$ values of the corresponding $\mathrm{pz}$ and $\mathrm{mpz}$ complexes are close to each other; the methyl substituent does not seem to influence the tetragonal distortion parameter very much.

iiii. The $D q^{z}$ values which can be obtained by the relation

$$
D q^{z}=D q^{x y}-\frac{7}{4} D t
$$


are much lower than the $D q$ values of the compounds $\mathrm{NiX}_{2}{ }^{23}$.

As concluded earlier, this is a manifestation of the unusually long metal-halide distance ${ }^{14-16}$.

\section{Experiments and Calculations Concerning the Ground State}

\section{A) Electron Paramagnetic Resonance}

Attempts were made to obtain EPR powder spectra for the compounds $\mathrm{Ni}(\mathrm{pz})_{4} \mathrm{X}_{2}$ and $\mathrm{Ni}(\mathrm{mpz})_{4} \mathrm{X}_{2}$. The compounds $\mathrm{Ni}(\mathrm{pz})_{4} \mathrm{X}_{2}$ showed no EPR spectra. This feature is expected for tetragonal compounds with large zero field splittings; the magnetic fields for obtaining such spectra should be higher than is experimentally available. For the compounds $\mathrm{Ni}(\mathrm{mpz})_{4} \mathrm{X}_{2}$ the zero field splitting is also large (vide infra). According to the structure determination ${ }^{16}$ these compounds have a symmetry lower than tetragonal. This lowering of symmetry with respect to the pyrazole compounds has also been detected in measurements on the powder isomorphic $\mathrm{Fe}(\mathrm{mpz})_{4} \mathrm{X}_{2}$ compounds ${ }^{24}$. By the action of the part of the Hamiltonian that reflects this lower symmetry the double degenerate ground state doublet $\Gamma_{5}\left(\mathrm{D}_{4 \mathrm{~h}}\right)$ is split. For a certain range of such splittings an EPR absorption at experimental magnetic fields is possible, due to a change of the energy level pattern with respect to the tetragonal case and, furthermore, mixing of the levels takes place so that now all possible transitions are allowed. Nevertheless, no EPR spectra were obtained for all three $\mathrm{Ni}(\mathrm{mpz})_{4} \mathrm{X}_{2}$ compounds, either at $\mathrm{X}$-band or at Q-band frequencies. Therefore, we can conclude that the splitting of $\Gamma_{5}$ is very small so that either the situation is too alike the tetragonal case or that the splitting of $\Gamma_{5}$ is so large that even no Q-band transitions are possible. We believe that because of the structural data the first situation is the more probable one.

\section{B) Magnetic Susceptibility}

For all three $\mathrm{Ni}(\mathrm{mpz})_{4} \mathrm{X}_{2}$ compounds magnetization measurements for varying magnetic fields at various temperatures were recorded as well as magnetic measurements for varying temperatures at a magnetic field of about 5000 Oersted. Calculations were carried out to fit these experimental data. We performed the calculations for purely paramagnetic compounds and used an ab initio method as given before $^{25}$. Moreover, a corrected method ${ }^{26,27}$ was applied to obtain the average magnetization of a powder. The spin Hamiltonian used in the calculations only involves tetragonal terms. This is because the lower symmetry splitting for the mpz compounds

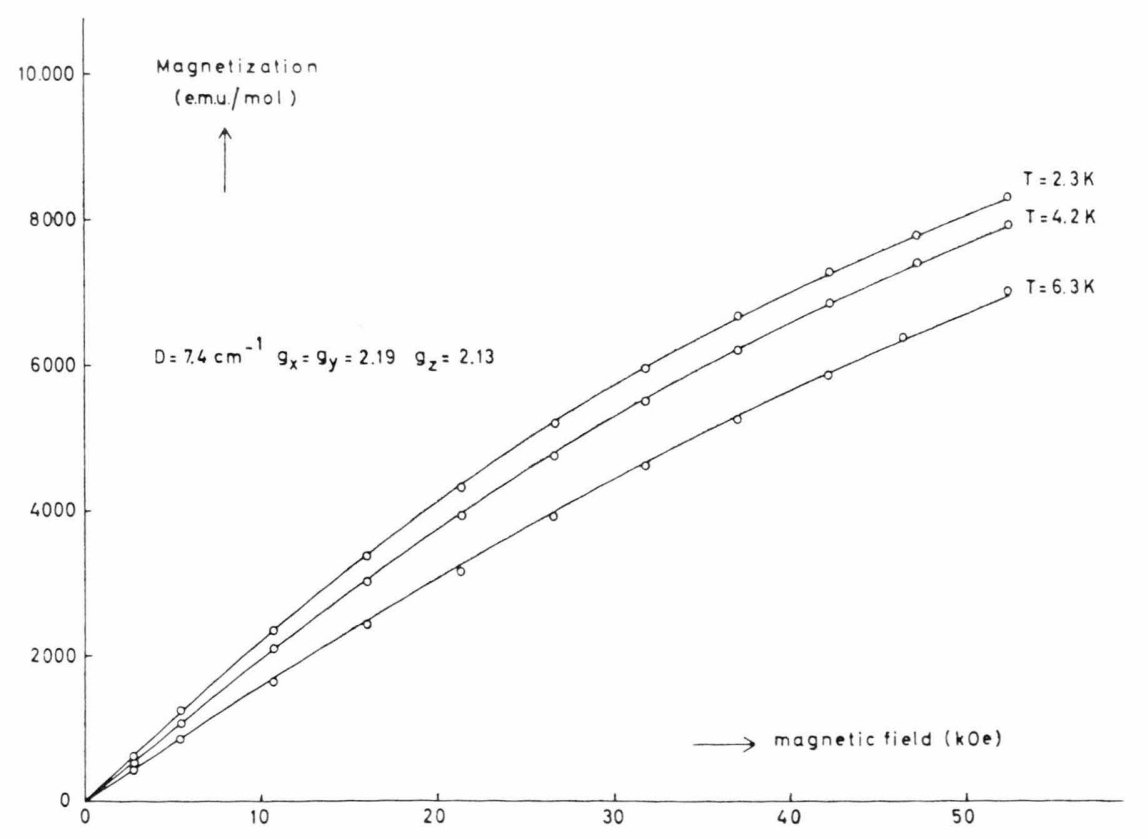

Fig. 1. Calculated saturation magnetization curves for $D=7.4 \mathrm{~cm}^{-1}, g_{x}=g_{y}=2.19$ and $g_{z}=2.13$ at temperatures of 2.3 , 4.2 and $6.3 \mathrm{~K}$. Measurements for $\mathrm{Ni}(\mathrm{mpz}){ }_{4} \mathrm{Cl}_{2}$ are shown as circles. 

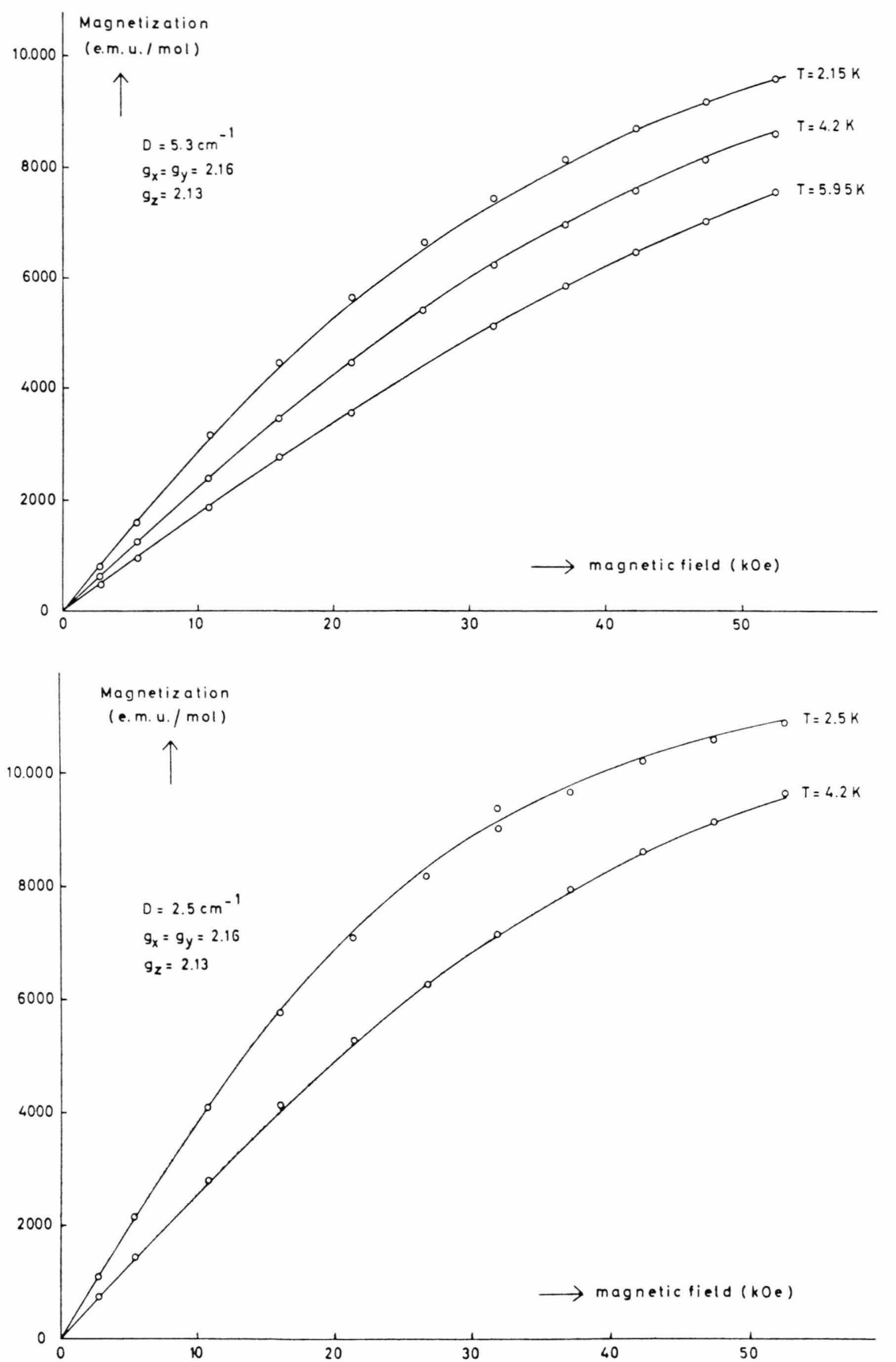

Fig. 2. Calculated saturation magnetization curves for $D=5.3 \mathrm{~cm}^{-1}, g_{x}=g_{y}=2.16$ and $g_{z}=2.13$ at temperatures of 2.15 , 4.2 and $5.95 \mathrm{~K}$. Measurements for $\mathrm{Ni}(\mathrm{mpz}){ }_{4} \mathrm{Br}_{2}$ are shown as circles.
Fig. 3. Calculated saturation magnetization curves for $D=2.5 \mathrm{~cm}^{-1}, g_{x}=g_{y}=2.16$ and $g_{z}=2.13$ at temperatures of 2.5 and $4.2 \mathrm{~K}$. Measurements for $\mathrm{Ni}(\mathrm{mpz}){ }_{4} \mathrm{I}_{2}$ are shown as circles. only influences the $\Gamma_{5}\left(\mathrm{D}_{4 \mathrm{~h}}\right)$ doublet which appears to be higher in energy (vide infra). It can be shown that the influence of this lower symmetry splitting on the magnetic behaviour will be negligible. In the calculations we used two kinds of $g$ values: $g_{\|}$, that is the $g$ value along the pseudo-tetragonal axis and $g_{\perp}$, the $g$ value perpendicular to that axis. From our experimental results these two $g$ values could not be determined separately. We, therefore, derived the average $g$ value from our high temperature magnetic data and next determined the distinct $g$ values by assuming that the orbital reduction factor $k$ is 

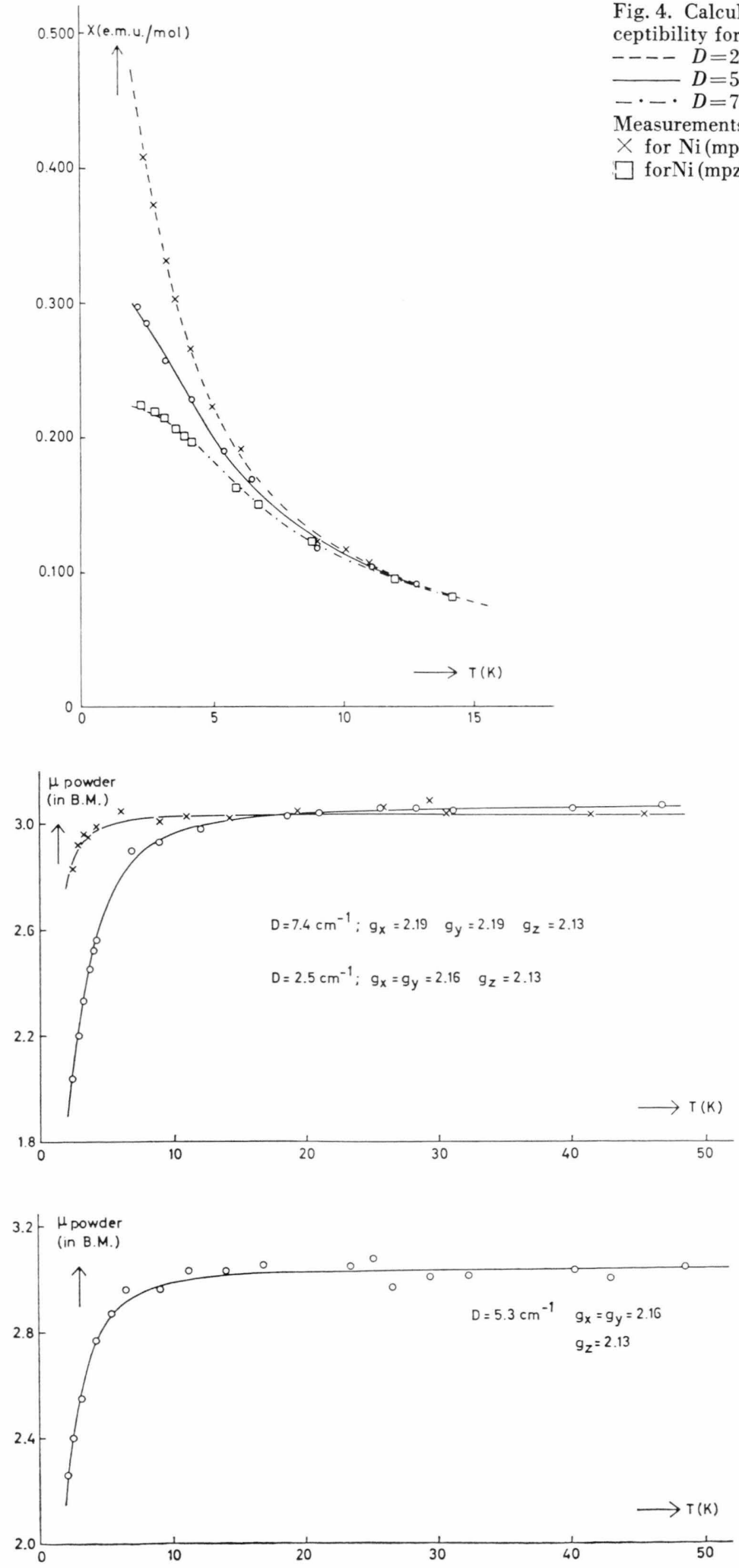

Fig. 4. Calculated curves for the powder paramagnetic susceptibility for

$$
\begin{aligned}
& -D=2.5 \mathrm{~cm}^{-1}, g_{x}=g_{y}=2.16, g_{z}=2.13, \\
& -D=5.3 \mathrm{~cm}^{-1}, g_{x}=g_{y}=2.16, g_{z}=2.13, \\
& -\cdot-\cdot D=7.4 \mathrm{~cm}^{-1}, g_{x}=g_{y}=2.19, g_{z}=2.13 .
\end{aligned}
$$

Measurements are shown as

$\times$ for $\mathrm{Ni}(\mathrm{mpz})_{4} \mathrm{I}_{2}, \bigcirc$ for $\mathrm{Ni}(\mathrm{mpz})_{4} \mathrm{Br}_{2}$,

$\square$ forNi(mpz) ${ }_{4} \mathrm{Cl}_{2}$.
Fig. 5. Calculated curves of powder paramagnetic moment for $D=2.5 \mathrm{~cm}^{-1}, g_{x}=g_{y}=2.16$ $g_{z}=2.13$ (the higher one at low temperature) and $D=7.4 \mathrm{~cm}^{-1}, g_{x}=g_{y}=2.19, g_{z}=2.13$ (the lower one at low temperature). Measurements are shown as $X$ for $\mathrm{Ni}(\mathrm{mpz})_{4} \mathrm{I}_{2}, \bigcirc$ for $\mathrm{Ni}(\mathrm{mpz}){ }_{4} \mathrm{Cl}_{2}$.

Fig. 6. Calculated curve of powder paramagnetic moment for $D=5.3 \mathrm{~cm}^{-1}, g_{x}=g_{y}=2.16$ and $g_{z}=2.13$. Measurements for $\mathrm{Ni}(\mathrm{mpz}){ }_{4} \mathrm{Br}_{2}$ are shown as circles. 
equal in the two distinct directions. For the calculation of the distinct $g$ values the ground state eigenfunctions were used, obtained by the ligand field calculations for the lower temperature. With the $g$ values determined in this way the zero field splitting was calculated by means of the results of magnetic measurements at low temperatures.

Table III. Ground state parameters of Ni(II) compounds.

\begin{tabular}{lllll}
\hline Compound & $D\left(\mathrm{~cm}^{-1}\right)$ & $g_{\perp}$ & $g_{\|}$ & $k$ \\
\hline $\mathrm{Ni}(\mathrm{mpz})_{4} \mathrm{Cl}_{2}$ & $7.4(2) \mathrm{a}$ & $2.13(2) \mathrm{b}$ & $2.19(2)$ & 0.85 \\
$\mathrm{Ni}(\mathrm{mpz})_{4} \mathrm{Br}_{2}$ & $5.3(2)$ & $2.13(2)$ & $2.16(2)$ & 0.65 \\
$\mathrm{Ni}(\mathrm{mpz})_{4} \mathrm{I}_{2}$ & $2.5(3)$ & $2.13(2)$ & $2.16(2)$ & 0.6 \\
$\mathrm{Ni}(\mathrm{pz})_{4} \mathrm{Cl}_{2}$ & $7.2(1)$ & $2.14(3)$ & $2.21(3)$ & \\
$\mathrm{Ni}(\mathrm{pz})_{4} \mathrm{Br}_{2}$ & $5.4(1)$ & $2.12(3)$ & $2.20(3)$ & \\
\hline
\end{tabular}

a Uncertainties in the last digit are shown in parentheses.

b The uncertainties shown for the $g$ values of the mpz compounds concern the average $g$ value.

The calculated curves as well as the experimental data are shown in Figures 1 to 6 . The two different types of magnetic measurements were fitted with the set of parameters $D, g_{\|}, g_{\perp}$ and $k$. In Table III the $D, g$ and $k$ values are listed for the compounds under investigation. The available data for two pyrazole compounds ${ }^{25}$ have been listed for comparison. Both the $D$ and $g$ values of $\mathrm{Ni}(\mathrm{mpz})_{4} \mathrm{X}_{2}$ are close to those of the corresponding $\mathrm{Ni}(\mathrm{pz})_{4} \mathrm{X}_{2}$ compounds; the differences being within experimental error.

Furthermore, calculations were carried out to explain the obtained $D$ values. We already calculated the crystal-field parameters and Racah parameters from our ligand-field data. For the following section the spectroscopic parameters obtained for the lower temperature were used. The $D$ values are, apart from the spectroscopic parameters also determined by the spin orbit coupling parameters $\zeta_{\|}$ and $\zeta_{\perp}$. To fit $D$ values with spin-orbit coupling parameters two different methods were followed:

1. Anisotropic spin-orbit interaction. Because it is assumed that the coordination perpendicular to the pseudo-tetragonal axis is equal for all three $\mathrm{Ni}(\mathrm{mpz})_{4} \mathrm{X}_{2}$ compounds, $\zeta_{\perp}$ was fixed and set at $\zeta_{\perp}=500 \mathrm{~cm}^{-1}$ introducing a reduction from the free ion value of $20 \%$. The $\zeta_{\|}$values were now chosen such that they were consistent with the $D$ value.
2. The spin-orbit coupling parameter was assumed to be isotropic and chosen to be consistent with the $D$ value. The spin-orbit coupling parameters that were obtained according to the two methods are shown in Table IV.

From Table IV it can be seen that the $\zeta$ values derived according to method 2 vary very much for the various anions. Especially the very low $\zeta$ value for $\mathrm{Ni}(\mathrm{mpz})_{4} \mathrm{I}_{2}$ seems to be unrealistic. In the past it has been shown that for some pseudo-tetragonal Ni compounds zero field splittings could be explained only by assuming an anisotropy in the spin-orbit coupling parameter ${ }^{26}$.

Table IV. Zero field splitting and spin-orbit coupling parameters of $\mathrm{Ni}$ (II) compounds (in $\mathrm{cm}^{-1}$ ).

\begin{tabular}{lllll}
\hline Compound & $D$ & \multicolumn{2}{l}{ Method 1 } & \multirow{2}{l}{ Method 2 } \\
\cline { 3 - 4 } & & $\zeta_{\|}$ & $\zeta_{\perp}$ & $\zeta$ \\
\hline $\mathrm{Ni}(\mathrm{mpz})_{4} \mathrm{Cl}_{2}$ & 7.4 & 500 & 460 & 582 \\
$\mathrm{Ni}(\mathrm{mpz})_{4} \mathrm{Br}_{2}$ & 5.3 & 500 & 522 & 438 \\
$\mathrm{Ni}(\mathrm{mpz})_{4} \mathrm{I}_{2}$ & 2.5 & 500 & 620 & 241 \\
$\mathrm{Ni}(\mathrm{pz})_{4} \mathrm{Cl}_{2}$ & 7.2 & 500 & 468 & 571 \\
$\mathrm{Ni}(\mathrm{pz})_{4} \mathrm{Br}_{2}$ & 5.4 & 500 & 540 & 419 \\
\hline
\end{tabular}

Also in our case the $\zeta$ values calculated according to method 1 seem to be more realistic than the $\zeta$ values according to method 2 . We remark that $\zeta_{\|}$ increases in the sequence $\mathrm{Cl}^{-}<\mathrm{Br}^{-}<\mathrm{I}^{-}$. The larger value of $\zeta_{\|}$in the halogen direction for $\mathrm{Br}^{-}$compared to $\mathrm{Cl}^{-}$was found before ${ }^{28}$.

\section{Conclusions}

From the results of our investigations the following conclusions may be drawn:

i. Both the ground-state splitting and the excitedstate splitting of these pseudo-tetragonal $\mathrm{Ni}$ (II) compounds can be fit with a single set of parameters.

ii. The zero-field splittings of the compounds $\mathrm{Ni}(\mathrm{mpz})_{4} \mathrm{X}_{2}$ closely resemble those of the corresponding pz compounds.

iii. The interpretation of the zero-field splittings using an anisotropic spin-orbit interaction shows that $\zeta$ in the halogen direction has the sequence $\mathrm{Cl}<\mathrm{Br}<\mathrm{I}$. 


\section{Acknowledgements}

The authors are indebted to Drs. H. T. Witteveen and M. C. Cadee for carrying out the magnetic measurements and to Drs. B. Nieuwenhuyse (University

1 C. J. Ballhausen, Introduction to ligand-field theory, McGraw-Hill, London 1962.

2 M. Gerloch, J. Lewis, and W. R. Smail, J. Chem. Soc. (A) 2434 [1971].

3 D. A. Rowley and R. S. Drago, Inorg. Chem. 7 (4), 795 [1968].

${ }^{4}$ R. L. Chiang and R. S. Drago, Inorg. Chem. 10 (3), 453 [1971].

5 J. R. Perumareddi, J. Phys. Chem. 79 (2), 142 [1975].

6 C. W. Reimann, J. Phys. Chem. 74 (3), 561 [1970].

7 J. Reedijk, Rec. Trav. Chim. 89, 605 [1970].

8 J. P. Fackler and D. G. Holah, Inorg. Chem. 4, 954 [1965].

9 A. H. M. Driessen-Fleur, Thesis, Leiden 1975.

10 R. D. Dowsing, B. Nieuwenhuyse, and J. Reedijk, Inorg. Chim. Acta 5, 301 [1971].

11 R. E. Trees, Phys. Rev. 83, 756 [1951] and 84, 1089 [1951].

12 G. Racah, Phys. Rev. 85, 381 [1952].

13 C. W. Ufford and H. B. Callen, Phys. Rev. 110, 1352 [1958].

14 C. W. Reimann, A. D. Mighell, and F. A. Mauer, Acta Cryst. 23, 135 [1967]. of Technology, Twente) and J. G. Vos for carrying out the EPR measurements. Prof. Dr. J. S. Wood (Amnerst, USA) is gratefully thanked for his valuable discussions.

15 A. D. Mighell, C. W. Reimann, and A. Santoro, Acta Cryst. B 25, 595 [1969].

16 J. Reedijk, B. A. Stork-Blaisse, and G. C. Verschoor, Inorg. Chem. 10, 2594 [1971].

17 M. T. Hutchings, Solid State Physics 16, 227 [1964].

18 T. Lulek, Phys. Stat. Sol. (b) 60, 439 [1973].

19 J. Reedijk, Rec. Trav. Chim. 89, 993 [1970].

20 J. R. Perumareddi, Z. Naturforsch. 27 a, 1820 [1972].

21 A. Mooney and W. E. Smith, J. C. S. Dalton 287 [1973].

22 J. Reedijk and D. W. Engelfriet, Rec. Trav. Chim. 91, 883 [1972].

23 M. Gerloch and R. C. Slade, Ligand-field parameters, Cambridge University Press, London 1973.

24 A. Vermaas, W. L. Groeneveld, and J. Reedijk, to be published.

25 F. W. Klaaijsen, J. Reedijk, and H. T. Witteveen, Z. Naturforsch. 27 a (10), 1532 [1972].

26 V. R. Marathe and S. Mitra, Chem. Phys. Letters 27 (1), 103 [1974].

27 A. Vermaas and W. L. Groeneveld, Chem. Phys. Letters 27 (4), 583 [1974].

28 F. W. Klaaijsen, Thesis, Leiden 1974. 